\title{
Chemical Constituents of Yarsagumba (Ophiocordyceps sinensis (Berk.) Sung et al.), a Valued Traditional Himalayan Medicine
}

\author{
Sabina Shrestha1, Bhushan Shrestha ${ }^{2,3}$, Ji-Hae Park ${ }^{1}$, Dae-Young Lee ${ }^{4}$, \\ Jin-Gyeong Cho ${ }^{1}$, and Nam-In Baek ${ }^{1}$ \\ ${ }^{1}$ Graduate School of Biotechnology, Institute of Life Sciences and Resources \\ Kyung Hee University, Yongin 446-701, Republic of Korea \\ ${ }^{2}$ Mushroom Research Division, National Institute of Horticultural and Herbal Science \\ Rural Development Administration, Suwon 441-707, Republic of Korea \\ ${ }^{3}$ Green Energy Mission/Nepal, Ghatte Kulo, Anam Nagar \\ Kathmandu, P.O. Box 10647 \\ ${ }^{4}$ Department of Medicinal Crop Research, National Institute of Horticultural and Herbal Science \\ Rural Development Administration, Eumseong 369-873 \\ Republic of Korea \\ e-mail:nibaek@khu.ac.kr
}

\begin{abstract}
Ophiocordyceps sinensis (Berk.) Sung et al. is popularly known as Yarsagumba (winter worm summer grass) in Nepal. It is a well-known entomogenous fungus distributed in alpine nival zone of trans-Himalayas and Tibetan Plateau. Its occurrence in extreme biological niche and cohabitation with insect [Thitarodes (Hepialus)] larvae has led to unique assemblage of metabolites including proteins and nitrogenous compounds, polysaccharides, sterols, nucleosides, fatty acids and their derivatives, vitamins and inorganics. In traditional Chinese medicine, it is one of the most trusted main ingredients for several preparations of remedy from wide range of human health conditions. Several biological activities from $O$. sinensis have been reported that include anti-inflammatory, antioxidant, antitumor, anti-metastatic, immunomodulatory, antimicrobial, insecticidal, hypolipidaemic, hypoglycemic, anti-ageing, lipolytic, neuroprotective, renoprotective effects, etc. The chemical constituents and their pharmacological uses are reviewed here highlighting the potentiality of this highly esteemed traditional Himalayan medicine.
\end{abstract}

Key words: Cordyceps sinensis, Ophiocordyceps sinensis, Thitarodes larvae, Yarsagumba

\section{Introduction}

Ophiocordyceps sinensis (Berk.) Sung et al. (Ophiocordicipitaceae, Hypocreales, Ascomycota, Kingdom Fungi) is a well-known entomogenous fungus distributed in alpine nival zone of transHimalayan terrain and Tibetan plateau in the altitudinal range of 3,000 to 5,000 asl. It has been commonly known by its synonym Cordyceps sinensis (Berk.) Sacc. both in scientific and non-scientific communities but has recently been transferred to new genus Ophiocordyceps on the basis of phylogenetic study (Sung et al. 2007). It is popularly known as Yarsagumba in Nepal, India and Bhutan, and is one of the mostprized herbs. In Ayurvedic literature, this herb was mentioned as Bhu-Sanjivani (Shrestha et al. 2010) and its uses were mentioned as Sannipatik Brikka Mahaphiranga, i.e., it cures severe and incurable kidney and syphilis diseases (Shrestha 2010, 2011). Other common Nepali names are Jivan Buti, Saram Buti, Kira Chhyau, Kira Jhar, Jingani, etc (Shrestha et al. 2010). The standard Chinese name is Dong Chong Xia Cao (Chong Cao in short), literally meaning winterworm summer-grass. Japanese and Korean names for this herb are Tochu-Kaso and Dong Chung Ha Cho respectively (Shrestha et al. 2010). The literal meaning of Yarsagumba in Tibetan language is coined on the basis of its appearance as a plant in the summer and an insect in the winter. It is regarded as highly effective herbal medicine for several diseases including cancer, hypoglycemia, asthma, hypercholesterolaemia, sexual 
dysfunction, immunodeficiency, etc (Zhu et al. 1998a,b). It is well known as tonic, aphrodisiac, cardiotonic and expectorant (Baral \& Kurmi 2006) and market value can even reach US\$ 800 for an ounce (Giove 2011). In Nepal, traditionally powdered material is taken with honey, milk or water. Alcoholic drink is also known to be prepared by immersing dry $O$. sinensis (Devkota 2006). It is also taken as an infusion with powdered root of Dactylorhiza hatagirea (Paanch aunle) or Ephedra gerardiana (Somlata) (Gewali 2008).

The scientific name for the sexual stage including stalked fruiting-body is termed as $O$. sinensis whereas its asexual mycelium culture is known as Hirsutella sinensis (Wei et al. 2011). In late summer, conidia or mycelia of the fungus invade and during winter eventually replace the internal organs of host larvae with thickened fungal tissue known as endosclerotium (Chen et al. 2004). When the temperature outside gets warm, fruiting-body (stroma) sprouts from the dorsal surface of prothorax of infected larvae, develops gradually and matures consequently in late summer (June-August) (Li \& Yang 2009). Wang \& Yao (2011) recognized nearly 60 taxa as potential hosts of $O$. sinensis. The host moths that mostly belong to genus Thitarodes (Hepialus) are commonly known as bat-moths, swift-moths or ghostmoths. Thitarodes larvae feed chiefly on soil humus and tender roots of Ranunculus brotherusii, Cyananthus macrocalyx, Juncus leucanthus and Veronica ciliate (Wei et al. 2011). The entire funguslarva combination is collected for medicinal use (Fig. 1). Its colour varies from light brown to brown or black which can be due to habitat difference as well as melanin concentration. Recently, Dong and Yao (2012), investigating on the presence of melanin in $O$. sinensis, emphasized that it may have role on strong endurance of $O$. sinensis to environmental stress. The growth of

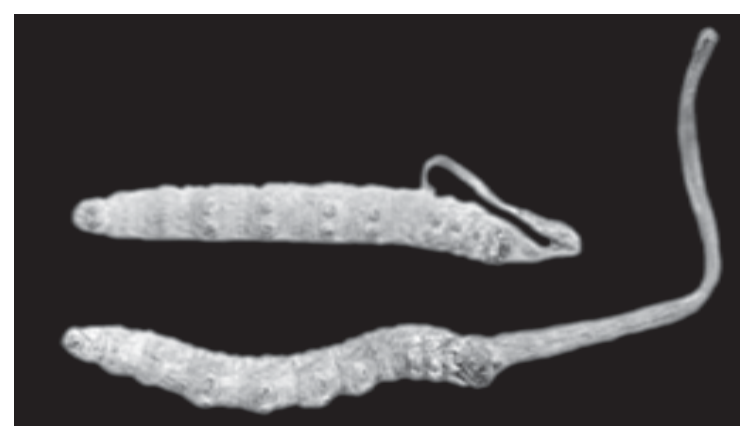

Fig. 1. Ophiocordycep sinensis from Manang. Courtesy of Himalayan Flowers, Trees and Animals (Shrestha et al. 2011).
O. sinensis has a very restricted habitat, and the yield is decreasing each year due to extensive harvest. Therefore, isolation of mycelium strains is done for large-scale culture and cultivation. The major constituents of $O$. sinensis and their pharmacological effects are dealt here to provide a concise insight into its potentiality as a time-honoured herb.

\section{Phytochemistry}

$O$. sinensis collected from wild is limited and expensive. Hence, cultured mycelium is choice for most of the studies. Phytochemical analysis of $O$. sinensis had led to isolation of proteins and nitrogenous compounds, polysaccharides, sterols, nucleosides, fatty acids and their derivatives, vitamins and inorganics (Zhu et al. 1998a,b, Holliday \& Cleaver 2008). Nucleosides are regarded as the active components and adenosine has been used as a marker for quality control of $C$. sinensis (Shiao et al. 1994). The chemical components such as ergosterol, adenosine, cordycepin, guanosine, inosine, uridine, mannitol and polysaccharides are major components of wild C. sinensis (Li et al. 2006). The inosine (33) content in natural $O$. sinensis contains much higher amount $(0.33 \mathrm{mg} / \mathrm{g})$ than culture ones (0.03-0.19) and cordycepin (27) content was also present in small quantity $(0.04 \mathrm{mg} / \mathrm{g})$ in wild but absent in cultured $O$. sinensis (Li et al. 2006). The highperformance liquid chromatography (HPLC) coupled with diode array detection (DAD) and evaporative light scattering detection (ELSD) method for qualitative and quantitative analysis showed carbohydrates, mannitol and trehalose are rich in $O$. sinensis in comparison to Cordyceps gunnii and C. liangshanensis and cultured Cordyceps mycelia. Further, uridine (34), adenosine (26) and guanosine (31) were the major components in cultured Cordyceps whereas cordycepin (27) was the major component in C. militaris (Wang et al. 2009). It is noteworthy that in LC/ESI-MS analysis $O$. sinensis had more adenine (23) content than $C$. militaris and hypoxanthine (25) was present in $O$. sinensis but not detected in C. militaris (Huang et al. 2003). Yang et al. (2011) isolated 50 compounds, including five new constituents, cordysinins A-E (39-43). They reported the presence of the significant amount of ergosterol (1), ergosteryl-3-O-â- $D$-glucopyranoside (2), $\hat{a}$ sitosterol (12), stigmasterol (15), uracil (22), $p$ hydroxybenzoic acid (81), 3-hydroxy-2-methyl-4pyrone (92), cyclo(L-Pro-L-Val) (65), cyclo(L-Pro-LTyr) (67), and D-mannitol (112) of in $n$-hexane/MeOH$\mathrm{H}_{2} \mathrm{O}$ and ethyl acetate fractions. Further, fifty-one volatile compounds including aldehydes, alcohols, 


\section{S. Shrestha et al./Chemical Constituents.......}

ketones, esters, aromatics, phenols, acids, alkanes, alkenes and the others were identified from the mycelia cultured by solid-state media and submerged fermentation, respectively (Yu et al. 2012). Previous research results have reported several pharmacological activities including improvement of physical performance, circulatory functions, atherosclerosis inhibition, respiratory system, kidney and renal system and anti-tumor, anti-metastatic (Holliday \& Cleaver 2008). The reported compounds are categorized here as isolated constituents (1-112) and detected volatile components (V1-V51) in Table 1.

Table 1. Major chemical constituents of Ophiocordyceps sinensis

Compounds
Sterols:
ergosterol (1), ergosteryl-3-O-â-D-glucopyranoside (2), ergosterol peroxide (3), 5á,8á-
epidioxy-24(R)-methylcholesta-6,22-dien-3â- D-glucopyranoside (4), (24R)-ergosta-7,22
diene-3â,5á,6â-triol (cerevisterol) (5), ergosta-4,6,8(14), 22-tetraen-3-one (6), 4,4-
dimethyl-5á-ergosta-8,24(28)-dien-3â-ol (7), 3-O-ferulylcycloartenol (8), 5á,6á-epoxy-
24(R)-methylcholesta-7,22-dien-3â-ol (9), ergosta-5-8(14),22-trien-7-one, 3â-ol [H1-A]
(10) 22,23-dihydroergosteryl-3-O-â-D-glucopyranoside (11), â-sitosterol (12), â-
sitosterol 3-O-acetate (13), daucosterol (14), stigmasterol (15), stigmasterol 3-O-acetate
(16), cholesterol (17), campesterol (18), dihydrobrassicasterol [D5-ergosterol] (19);
fungisterol [D-ergosterol] (20),(17R)-17-methylincisterol (21)

\section{Nitrogenous compounds:}

uracil (22), adenine (23), guanine (24), hypoxanthine (25), adenosine (26), cordycepin (27), dideoxyadenosine (28), $\mathrm{N}^{6}$-(2-hydroxyethyl) adenosine (29) inosine (30), guanosine (31), thymine (32), thymidine (33), uridine (34), dideoxyuridine (35), cordyceamides A (36), cordyceamides B (37), aurantiamide acetate (38), cordysinin A (39), cordysinin B (40), cordysinin C (41), cordysinin D (42), cordysinin E (43), cordycedipeptide A (3acetamino-6-isobutyl-2,5-dioxopiperazine) (44), 3-isopropyl-6-isobutyl-2,5dioxopiperazine (45), 3,6-di(4-hydroxy)benzyl-2,5-dioxopiperazine (46), caffeine (47), $\mathrm{N}$-(2'-hydroxy-tetracosanoyl)-2-amino-1,3,4-trihydroxy-octadec-8E-ene (tetracosanamide) (48), 2-nicotinic acid (49)

Proteins: nucleic acids, amino acids, polyamines

cyclo-(Gly-Pro) (50), cyclo-(Leu-Pro) (51), cyclo-(Val-Pro) (52), cyclo-(Ala-Leu) (53), cyclo-(Ala-Val) (54), and cyclo-(Thr-Leu) (55), 1,3-diamino propane (56), cadaverine (57), spermidine (58), spermine (59), putrescine (60), flazin (61), perlolyrine (62), 1methylpyrimidine-2,4-dione (63), 1-acetyl-â-carboline (64), cyclo(L-Pro3-L-Val) (65), cyclo(L-Phe-L-Pro) (66), cyclo(L-Pro-L-Tyr) (67), cordymin (68), L-tryptophan (69)

\section{Fatty acids and other organic acids:}

palmitic acid (70), lauric acid (71), myristic acid (72), pentadecanoic acid (73), palmitoleic acid (74), linoleic acid (75), oleic acid (76), stearic acid (77), docosanoic acid (78), lignoceric acid (79), succinic acid (80)

Phenolics and acids:

p-hydroxybenzoic acid (81), vanillic acid (82), syringic acid (83), p-methoxybenzoic acid (84), $p$-hydroxyphenylacetic acid (85), 3,4-dihydroxyacetophenone (86), 4 hydroxyacetophenone (87), protocatechuic acid (88), 3, p-methoxyphenol (89), acetovanillone (90), salicylic acid (91), 3-hydroxy-2-methyl-4-pyrone (92), methyl p-hydroxyphenylacetate (93), 2-deoxy-D-ribono-1,4-lactone (94), furancarboxylic acid (95)

\section{Isoflavones:}

$32,42,7$-trihydroxyisoflavone (96), glycitein (97), daidzein (98), orobol (99), genistein

Yang et al. 2011 (96-100)

\section{Reference}

Kadota et al. $1986(\mathbf{1 7}, \mathbf{1 8}, \mathbf{1 9})$;

Bok et al. 1999 (2, 3, 4, 9, 11);

Lin et al. 1999 (10); Li et al. 2003

$(\mathbf{1}, 3,5,12,17)$; Yang et al. 2011

$(6-8,12-16,20-21)$

Zhu et al. 1998a (22, 31, 33-35);

Huang et al. 2003 (23, 25-27);

Li et al. 2003 (47,48); Jia et al.

2005 (44-46); Li et al. 2006 (28-31,

34); Holliday \& Cleaver 2008 (26-

29); Jia et al. 2009 (36-38); Liu et

al. 2010 (22-26, 30, 34); Yang et al.

2011 (32, 39-43, 49)

Zhang et al. 1991 (69); Holliday

\& Cleaver 2008 (50-60), Yang et

al. 2011 (61-67); Qian 2012 (68)

Li et al. 2003 (70); Yang et al. 2009 (70-79) Yang et al. 2011 (80)

Yang et al. 2011 (81-95) (100) 


\section{Polysaccharide and sugar derivatives:}

CS-F30 [Gal:Glc:Man = 62:28:10](101), CS-F10 [Gal:Glc:Man = 43:33:24] (102), CT-4N [Man:Gal =3:5] (103), CS-81002 [Man:Gal:Glc = 10.3:3.6:6.1] (104), SCP-I [D-glucan](105), CSP-1 [GlC:Man:Gal = 1:0.6:0.75] (106), CPS1 [Glc:Man:Gal = 2.8:2.9:1] (107), cordysinocan [Glc:Man:Gal = 2.4:2.1] (108), PS-A [Glc:Gal:Man = 2:1:1] (109), CS-PS [Man:Rhm:Ara:Xyl:Glc:Gal = 38.37:2.51:2.21:5.22:27.44:24.45] (110), mannoglucan [Man:Glc = 1:9] (111), Dmannitol (112)
Kiho et al. 1986 (103); Gong et al. 1990 (104); Kiho et al. 1993 (101); Kiho et al. 1999 (102); Li et al. 2003 (106); Wu et al. 2006 (105); Wu et al. 2007 (111); Cheung et al. 2009 (108); Wang et al. 2009 (107); Kim 2010 (109); Zhang et al. 2011 (110); Yang et al. 2011 (112)

\section{Vitamins:}

B1, B2, B12, E, and K

Zhu et al. 1998a

Inorganics:

K, Na, Ca, Mg, Fe, Cu, Mn, Zn, Pi, Se, Al, Si, Ni, Sr, Ti, Cr, Ga, V, and Z Zhu et al. 1998a

\section{Volatile compounds:}

Aldehydes: Benzaldehyde (V1), benzeneacetaldehyde (V2), (E)-2-dodecenal (V3), nonanal (V4), (E)-2-Nonenal (V5), 4-fluoro-3-hydroxy-benzaldehyde (V6), decanal (V7), (E,E)-2,4-nonadienal (V8);

Alcohols: phenylethyl alcohol (V9), 2-(methylthio)-3-pyridinol (V10), p-menth-

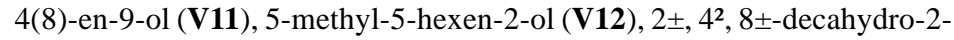
naphthalenol(V13), 2-Butyl-2,7-octadien-1-ol (V14), 4,6-di-tert-butyl-m-cresol (V15);

Ketones: 6-ethenyldihydro-2,2, 6-trimethyl-2H-pyran-3(4H)-one (V16), trans-3nonen-2-one (V17), 1-oxaspiro(4,5)decan-2-one (V18), 4-(2-furanyl)-3-buten-2one (V19), 7-chloro-2,3-dihydro-3-(4-N,N-dimethylaminobenzylidene)-5-phenyl1H-1,4-benzodiazepin-2-one(V20), 1,5-dihydro-1-methyl-2H-pyrrol-2-one (V21), (Z)-dihydro-5-(2-octenyl)-2(3H)-furanone (V22), 4-butoxy-3-penten-2one (V23);

Esters: 2-butynedioic acid, di-2-propenyl ester (V24), 1,3-cyclohexadiene-1,3diol, 5,5-dimethyl-diacetate (V25), benzoic acid, 2,4-bis[(trimethylsilyl)oxy]trimethylsilyl ester (V26), oxalic acid, isobutyl tetradecyl ester (V27);

Aromatics: azulene (V28), 1-methylene-1H-indene (V29), 2,6-dimethylnaphthalene (V30), 1,6-dimethyl-naphthalene (V31);

Phenols: 2-methyl-phenol (V32), butylated hydroxytoluene (V33);

Acids: phosphonic acid (V34), alkanes: decamethyl-cyclopentasiloxane (V35), 1chloro-nonadecane (V36), 2,4-diisopropyl-1,1-dimethyl-cyclohexane (V37), dodecamethyl-cyclohexasiloxane, (V38), 2-methyl-dodecane (V39), tetradecamethyl-cycloheptasiloxane (V40), 2,6,10,14-Tetramethyl-hexadecane (V41), 1,54-Dibromo-tetrapentacontane (V42);

Alkenes: (E)-9-eicosene (V43);

Others: 2-pentyl-furan (V44), 2-hexyl-2,4-decadienal, (E,E)-furan(V45), methyl 2,3-anhydro-4-azido-4-deoxy-2-L-Ribopyranoside (V46), 1,2-benzisothiazole (V47), indole (V48), 1-(chloromethyl)-3-methoxy-benzene (V49), 1,2benzisothiazole, 3-(hexahydro-1H-azepin-1-yl)-1,1-dioxide (V50), disulfide, ditert-dodecyl (V51) 


\section{Toxicity}

There are very few reports of toxic side effects like dry mouth, nausea or diarrhea (Zhu et al. 1988a,b). Rabbit given $10 \mathrm{~g} / \mathrm{kg}$ per day $(\mathrm{n}=6)$ did not show any abnormalities in blood test or in kidney or liver function (Huang et al. 1987). Further, for median lethal does $\left(\mathrm{LD}_{50}\right)$, mice injected up to $80 \mathrm{~g} / \mathrm{kg}$ per day, did not show any fatalities after 7 days (Holliday et al. 2005). However, there is a report of a case of systemic allergic reaction after taking a strain of Cs-4 (Huang et al. 1987). Lie et al. (2005) observed non toxic nature $O$. sinensis extract in test for animal mass, biochemical properties of blood, and histopathological changes of liver and kidney. Further, test for acute toxicity in bone marrow chromosome aberrations and Ames test also showed it as safe (Shen et al. 2001). However, published data on effect on children as well as pregnant or lactating women is still lacking.

The adulteration of wild harvest is one of the major problems in trade of $O$. sinensis. The methodology to ensure authenticity and quality of wild $O$. sinensis and its products for safety and pharmacological efficacy is appearing as outmost importance. However, there are still drawbacks in use of multiple markers such as nucleosides, ergosterol, mannitol and polysaccharides used for quality control (Li et al. 2006). Fu et al. (2011) reported process for more precise authentication of sample continuous monitoring of cellular impedance in real time, which produces specific time/dose dependent cell response profiles (TCRPs) in addition to spectroscopic fingerprinting of active compounds by HPLC using adenosine (or 3-deoxyadenosine) as a standard, in accordance with Pharmacopoeia of the People's Republic of China (2005 version).

\section{Chemical Constituents}

Li et al. (2002) showed observed resemblance and pharmacological efficacy of nucleoside and polysaccharide contents in fruiting body and caterpillar host by capillary electrophoresis (CE) and fast performance liquid chromatography (FPLC) analysis. It is noteworthy that the CE profile of the dry naive worm without Cordyceps invasion was completely different. Natural O. sinensis contains more than $7.99 \%$ free mannitol, whereas cultured $O$. sinensis contained less than 5.83\% mannitol (Guan et al. 2010). Zhu et al. (1998a) classified seven classes of chemical constituents in natural and mycelium fermentation of $O$. sinensis as sterols, nucleoside compounds, saccharides and sugar derivatives, fatty acids and other organic acids, proteins, vitamins and inorganics. The constituents of $O$. sinensis are numerous and vary depending on the place of origin and type of strain used for mycelium culture. It is not our intention in this review to cover all compounds reported, but to summarize the major components (compounds 1-112). Several characteristic compounds have been reported from $O$. sinensis (Fig. 2).<smiles>[R6][C@H]1CC[C@]2(C)C(=CC=C3C2CC[C@]2(C)[C@H]3CC[C@@H]2[C@H](C)/C=C/[C@@H](C)C(C)C)C1</smiles>

R

H (1) ergosterol

Glu (2) ergosteryl-3-O-â-D-glucopyranoside<smiles>[R20][C@H]1CC[C@@]2(C)[C@@H]3CC[C@]4(C)[C@@H]([C@H](C)/C=C/[C@H](C)CC)CC[C@H]4[C@]34C=C[C@@]2(C1)O4</smiles>

R

H (3) ergosterol peroxide

[5á,8á-epidioxy-24(R)-methylcholesta-6,22-diene-3âol]

Glu (4) 5á,8á-epidioxy-24(R)-methylcholesta-6,22-dien3â-O-â-D-glucopyranoside<smiles>CC(C)[C@H](C)/C=C/[C@@H](C)[C@H]1CC[C@H]2C3=C[C@H](O)[C@@]4(O)C[C@@H](O)CC[C@]4(C)[C@@]3(C)CC[C@@]21C</smiles>

(5) cerevisterol

(24R)-ergosta-7,22-diene-3â,5á,6â-triol 


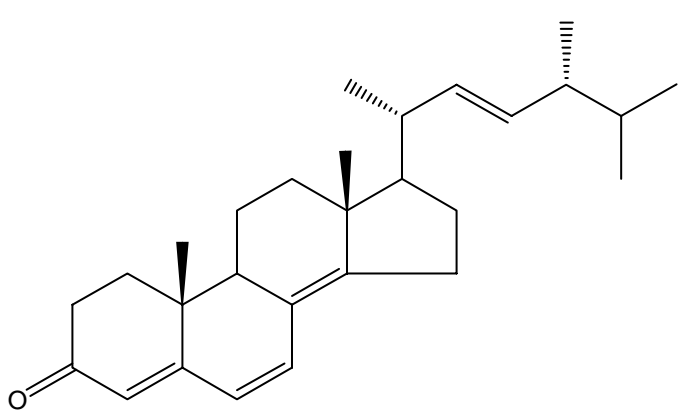

(6) ergosta-4,6,8(14),22-tetraen-3-one<smiles>C=C(CC[C@@H](C)C1CCC2C3=C(CC[C@]21C)[C@@]1(C)CC[C@H](O)C(C)(C)C1CC3)C(C)C</smiles>

(7) 4,4-dimethyl-5á-ergosta-8,24(28)-dien-3â-ol<smiles>C=CCC[C@H](C)C1CCC2[C@H]3CCC4C(C)(C)[C@@H](OC(=O)/C=C/c5ccc(O)c(OC)c5)CCC45C[C@]35CC[C@@]12C</smiles>

(8) 3-O-ferulylcycloartenol<smiles></smiles>

(9) 5á,6á-epoxy-24(R)-methylcholesta-7,22-dien-3â-ol<smiles>CC(C)[C@H](C)/C=C/[C@H](C)[C@H]1CCC2=C3C(=O)C=C4C[C@@H](O)CC[C@]4(C)C3CC[C@]21C</smiles>

(10) ergosta-5,8(14),22-trien-7-one, 3â-ol [H1-A]

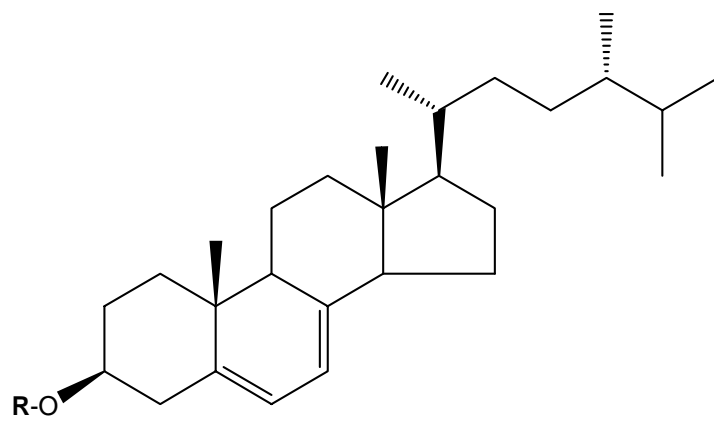

(11) 22,23-dihydroergosteryl-3-O-â-D-glucopyranoside<smiles>[R]O[C@H]1CC[C@]2(C)C(=CC[C@H]3[C@@H]2CC[C@@]2(C)[C@H]([C@H](C)CC[C@H](CC)C(C)C)CC[C@@H]32)C1</smiles>

Glu (14) daucosterol

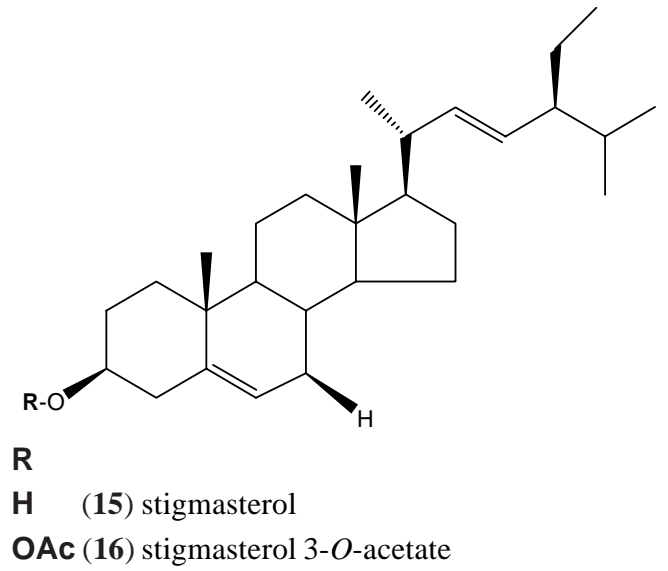


S. Shrestha et al./Chemical Constituents.......

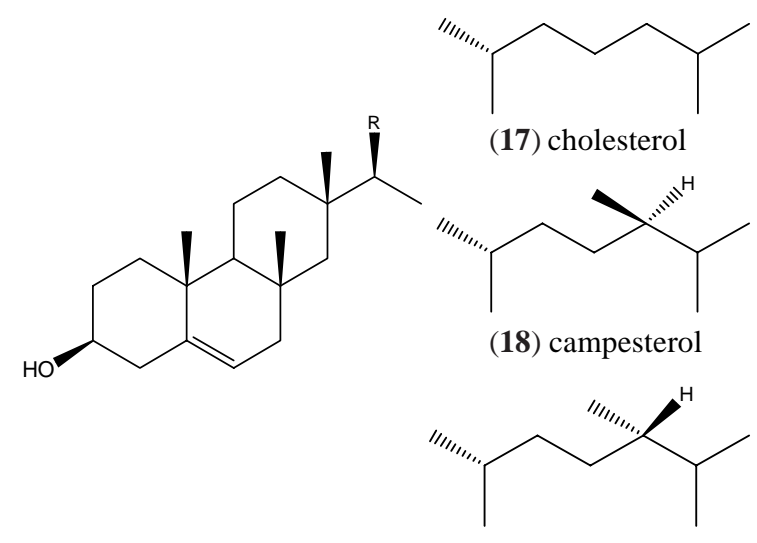

(19) dihydrobrassicasterol ( $\Delta^{5}$-ergosterol)
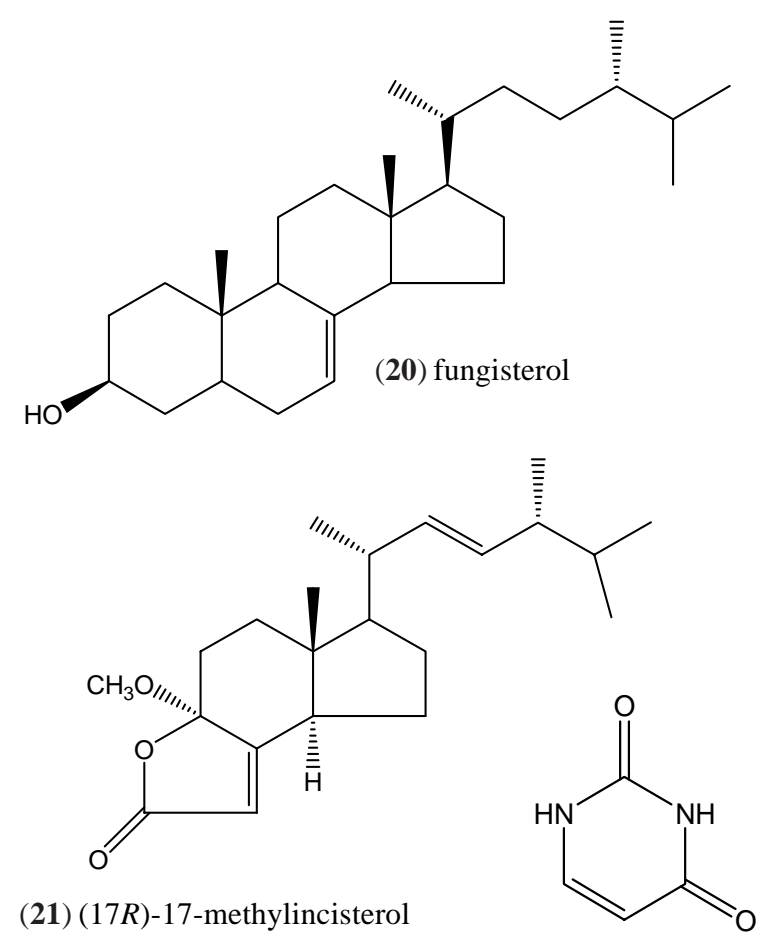

(22) uracil<smiles>[R]c1ncnc2[nH]cnc12</smiles>

$\mathbf{R}$

$\mathrm{NH}_{2}$ (23) adenine

$\mathrm{OH}$ (25) hypoxanthine<smiles>[R]n1cnc2c(=O)[nH]c(N)nc21</smiles>

Ribose (31) guanosine<smiles></smiles>

$\begin{array}{ll}\mathbf{R}_{1} & \mathbf{R}_{2}\end{array}$

$\mathrm{OH} \mathrm{OH}(26)$ adenosine

H OH (27) cordycepin

H H (28) dideoxyadenosine<smiles>O=c1[nH]cnc2c1ncn2[C@@H]1O[C@H](CO)[C@H](O)[C@H]1O</smiles><smiles>OC[NH2+]c1ncnc2c1ncn2[C@@H]1O[C@H](CO)[C@H](O)[C@H]1O</smiles>

(29) $\mathrm{N}^{6}$-(2-hydroxyethyl) adenosine

(30) inosine<smiles>Cc1cn([C@H]2C[C@H](O)[C@@H](CO)O2)c(=O)[nH]c1=O</smiles><smiles></smiles>

$\mathrm{OH}$ (34) uridine

H (35) deoxyuridine<smiles>Cc1c[nH]c(=O)[nH]c1=O</smiles>

(32) thymine 
Nepal Journal of Science and Technology Vol. 13, No. 1 (2012) 43-58<smiles>[R]c1ccc(CC(COC(C)=O)NC(=O)C(Cc2ccc([R])cc2)NC(=O)c2ccccc2)cc1</smiles>

$\begin{array}{ll}\mathbf{R}_{1} & \mathbf{R}_{2} \\ & \end{array}$

OH H (36) cordyceamide A [N-benzoyl-L-tyrosinylL-phenylalaninol acetate]

OH OH (37) cordyceamide B [ $N$-benzoyl-L-tyrosinyl-Lp-hydroxyphenylalaninol acetate]

H $\quad$ H (38) aurantiamide acetate

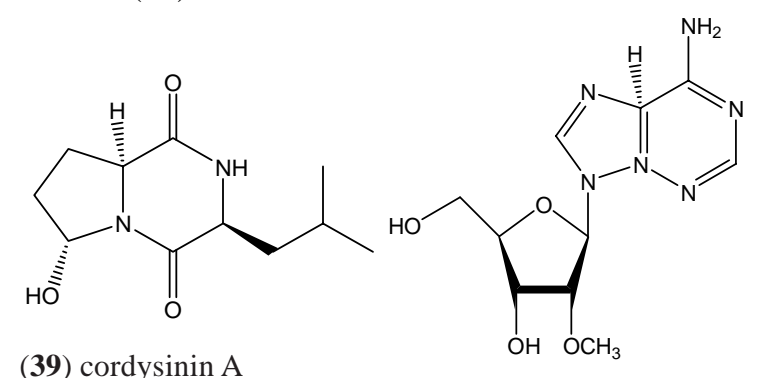

(40) cordysinin B

$\mathbf{R}$<smiles>[R]c1nccc2c1[nH]c1ccccc12</smiles><smiles>CC(C)O</smiles>

(41) cordysinin C

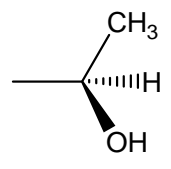

(42) cordysinin D<smiles>CC[C@H](O)CO</smiles>

(43) cordysinin E<smiles>[R][C@H]1NC(=O)[C@@H]([C@H](C)CC)NC1=O</smiles><smiles>[R]C(=O)CC(N)=O</smiles>

(44) cordycedipeptide A

[3-acetamino-6-isobutyl-2,5-dioxopiperazine]

(45) 3-isopropyl-6-isobutyl-2,5-dioxopiperazine<smiles>O=C1NC(Cc2ccc(O)cc2)C(=O)NC1Cc1ccc(O)cc1</smiles>

(46) 3,6-di(4-hydroxy)benzyl-2,5-dioxopiperazine<smiles>Cn1c(=O)c2c(ncn2C)n(C)c1=O</smiles>

(47) caffeine<smiles>CCC=CCC(O)C(O)C(CO)NC(=O)C(O)CC</smiles>

(48) $N$-(2'-hydroxy-tetracosanoyl)-2-amino-1,3,4trihydroxy-octadec-8E-ene<smiles>O=C(O)c1cccnc1</smiles>

(49) 2-nicotinic acid

Fig. 2. Chemical structures of characteristic compounds (1-49) from O. sinensis 


\section{S. Shrestha et al./Chemical Constituents.......}

Some new compounds recently reported from mycelium of $O$. sinensis are cordycedipeptide A [3-acetamino-6isobutyl-2,5-dioxopiperazine, 3-isopropyl-6-isobutyl2,5-dioxopiperazine 3,6-di(4-hydroxy) benzyl-2,5dioxopiperazine] (44) (Jia et al. 2005), cordyceamides A and B (Jia et at. 2009) and cordysinins A-E (39-43) (Yang et al. 2011). Several polysaccharides have been isolated from O. sinensis (Zhong et al. 2009, Wu et al. 2006). Further, 51 volatile compounds (V1-V51) were characterized from the mycelia of $O$. sinensis (Yu et al. 2012). Among them phenols, acids and alkanes were found as the major classes of compounds. Among the total volatiles, butylated hydroxytoluene (BHT) (V33) was it is noteworthy that the most abundant volatile compound $47.38 \%$ and $46.12 \%$ in solid state media and submerged fermentation, respectively. There is very few report of presence of BHT in natural source (Babu \& Wu 2008). Phosphonic acid (V34) was the second abundant compounds and accounted for $24.96 \%$ and $7.01 \%$ in mycelia cultured by submerged fermentation and mycelia cultured by solid-state media, respectively.

\section{Pharmacological reports}

In traditional medicine practices, the therapeutic effects are due to contribution of multiple components, not only the major ones. There exist extensive reviews on clinical aspects of $O$. sinensis (Zhu et al. 1998a,b, Holliday 2008, Li 2009). Here we have dealt with an overview of therapeutic values of major compounds present in $O$. sinensis and their pharmacological significances.

\section{Cytotoxicity}

There are reports of water, ethanol extract and polysaccharide fractions of $O$. sinensis attributing immune response rather than direct cytotoxicity (Wasser 2002, Nakamura et al. 2003, Yoshida et al. 1989). Zhao et al. (2011) reported ergosta-4,6,8(14),22tetraen-3-one (6) induces G2/M cell cycle arrest and apoptosis in HepG2 cells in a caspase-dependent manner. Further the interference of altered nucleoside like cordycepin (27) can have a role in DNA repair mechanism for antitumor response (Holliday \& Cleaver 2008). Jia et al. (2005, 2009) reported cordyceamides A (36) and B (37) as well as cordycedipeptide A (44) as cytotoxic against L-929, A375 and Hela IC50. Cordycedipeptide $A(44)$ showed significant $\mathrm{IC}_{50}$ value of $6.30 \mathrm{mg} / \mathrm{ml}$ (L-929), $9.16 \mathrm{mg} / \mathrm{ml}$ (A375). Moreover, the glycosylated form of ergosterol peroxide (4) as a greater inhibitor to the proliferation of K562, Jurkat,
WM-1341, HL-60 and RPMI-8226 tumor cell lines by 10 to $40 \%$ at $10 \mathrm{ig} / \mathrm{ml}$ (Bok et al. 1999). The presence of above mentioned potential cytotoxic compounds might be responsible for anticancer property of $O$. sinensis.

\section{Anti-inflammation}

There are several reports of anti inflammatory property from extract of $O$. sinensis (Rao et al. 2007, Shahed et al. 2001, Kuo et al. 2001, Diniz et al. 2003, Jordan \& Lee 2008). Kim et al. (2006) reported that cordycepin (27) markedly inhibited the phosphorylation of Akt and p38 in dose-dependent manners in LPS-activated macrophage. It suppressed tumor necrosis factor (TNF-á) expression, IêB alpha phosphorylation, and translocation of nuclear factor-êB (NF-êB). The expressions of cycloxygenase-2 (COX-2) and inducible nitric oxide synthase (iNOS) were significantly decreased in RAW 264.7 cell by cordycepin (27). These results suggest that cordycepin (27) inhibits the production of $\mathrm{NO}$ production by down-regulation of iNOS and COX-2 gene expression via the suppression of NF-êB activation, Akt and p38 phosphorylation. Further, Yang et al. (2011) reported ergosterol-3-O-â-D-glucopyranoside (2) displayed the most significant inhibition of superoxide anion generation and elastase release with $\mathrm{IC}_{50}$ values of $5.42 \pm 0.50$ and $5.62 \pm 0.37 \mathrm{iM}$, respectively. They also reported 1-(5-hydroxymethyl-2-furyl)-â-carboline (perlolyrine) (62) displayed the most significant inhibition of superoxide anion generation and elastase release with $\mathrm{IC}_{50}$ values of $0.45 \pm 0.15$ and $1.68 \pm 0.32$ ìM, respectively. Thus, cordycepin (27), ergosterol-3$O$-â-D-glucopyranoside (2) and perlolyrine (62) may have provide a significant role in treatment of inflammation-associated disorders.

\section{Antioxidant \\ Xiao et al. (2012) reported cordycepin (27) could ameliorate albumin-induced EMT of HK2 cells by decreasing NADPH oxidase activity and inhibiting reactive Oxygen Species (ROS) production. A heteropolysaccharide, CS-PS have been reported for its effect on immunity activity in mice treated by ionizing radiation, through reducing oxidative injury and modulating the secretion of cytokine IL-4, IL-5 and IL-17 (Zhang et al. 2011). Wang et al. (2012) reported cordymin (68) significantly enhanced the defense mechanism against cerebral ischemia by increasing antioxidants activity related to lesion pathogenesis leading the brain recover from ischemic injury. Yang et al. (2011) observed ergosteryl-3-O-â-}


D-glucopyranoside (2) and perlolyrine (62) inhibited superoxide anion generation and elastase release in FMLP/CB-activated human neutrophils. They reported that the most significant inhibition toward superoxide anion generation and elastase release with $\mathrm{IC}_{50}$ values of $0.45 \pm 0.15$ and $1.68 \pm 0.32 \mathrm{iM}$, respectively were demonstrated by perlolyrine. Further, compound 3',4',7trihydroxyisoflavone (96) displayed significant scavenging of DPPH free radicals with $\mathrm{IC}_{50}$ values of 31.97 ìM. Therefore, antioxidant activity may be attributed to the presence of ergosteryl-3-O- $\hat{a}-\mathrm{D}$ glucopyranoside (2), perlolyrine (62) and 3',4',7trihydroxyisoflavone (96). Polysaccharide CPS1 (107) provided scavenging effects on the hydroxyl radicals, the reducing power, $\mathrm{Fe}^{2+}$-chelating activity, scavenging effect on superoxide radicals, antioxidant activity and showed a connection between antioxidant activity and reparation of renal failure (Wang et al. 2009). The polysaccharide CSP-1 (106) is known for significant protection against the free radical-induced neuronal cell toxicity by attenuating the changes of glutathione peroxidase and superoxide dismutase activities in $\mathrm{H}_{2} \mathrm{O}_{2}$-treated cells in a dose-dependent manner ( $\mathrm{Li}$ et al. 2003).

\section{Immunomodulating effect}

The renal failure is one of the major human health issues and improvement of renal function by use of O. sinensis is reported (Guan et al. 1992). Xu et al. (1995) reported immunomodulating effects of $O$. sinensis in kidney transplanted patients. The lymphoproliferative response, natural killer (NK) cell activity, and phytohemagglutinin (PHA) stimulated interleukin-2 (IL-2) and tumor necrosis factor-alpha (TNF-alpha) production on human mononuclear cells (HMNC) (Kuo et al. 1996). Lin et al. (1999) observed H1-A (10) can suppress the activated human mesangial cells HMC and alleviate IgAN (Berger's disease) with clinical and histological improvement. Ergosterol (1) is well known as diuretic bioactive compound with excellent efficacy (Zhao 2009). Ding et al. (2011) reported lowering of serum level of IL-10 in kidney transplanted patient, which can be beneficial in long term treatment requiring cyclosporin. Lin (2002) reported (24R)-ergosta-7,22-dien-3â,5á,6âtriol (5) improved kidney function in renal diseases, including IgA nephritis, autoimmune nephritis, and lupus nephritis by inhibiting IL-2 formation by monocyte and proliferation of mesangial cells and lymph node.

\section{Hyperglycemia}

Guo and Zhang (1995) reported potentiality of $O$. sinensis in blood sugar regulation. Kiho et al. (1996) reported CS-F30 (101) increased the activities of hepatic glucokinase, hexokinase and glucose-6phosphate dehydrogenase and decreased glycogen content in the liver. Furthermore, Kiho et al. (1999) reported CS-F10 (102) significantly increased the activity of hepatic glucokinase in streptozotocin (STZ) -induced diabetic mice leading to reduced hepatic glucose output. Li et al. (2006) reported CSP-1 (106) a strong antioxidant showed with hypoglycemic properties increased circulating insulin level in diabetic animals, suggested that CSP-1 (106) may stimulate pancreatic release of insulin and/or reduce insulin metabolism. Yun et al. (2003) reported cordycepin (27) can reduced blood glucose level by $35.5 \%$ (acarbose, $37.2 \%$ ) in STZ induced diabetic mice and elaborated that it can be promising new drug as an antihyperglycemic agent without defects of immune responses and the other side effects.

Asthemia

O. sinensis is known for its beneficial effect on various lung ailments (Holliday and Cleaver 2008) as well as in severe acute respiratory syndrome (SARS) (Chen et al., 2012). Yue et al. (2008) reported cordycepin and adenosine stimulated ion transport in a dosedependent manner in Calu-3 monolayers and which may be responsible for anion movement from the basolateral to apical compartments in the airway epithelia. Basolateral $\mathrm{Na}^{+}-\mathrm{K}^{+}-2 \mathrm{Cl}^{-}$cotransporter and apical cAMP-dependent cystic fibrosis transmembrane conductance regulator $\mathrm{Cl}^{-}$channel are involved in the process leading to pharmacological effect on the respiratory tract. Lin et al. (2004) reported use of (24R)ergosta-7,22-diene-3â,5á,6â-triol (5) for preventing and treating bronchial hyper responsiveness and acute asthma attack and improving pulmonary function.

\section{Heart ailment (arrhythmias, cardiac arrest)}

Jordan et al. (2008) reported use of $O$ sinensis to reduce acute and chronic rejection associated with cardiac transplantation. The use of $O$. sinensis extract in conjunction with a sub-therapeutic dose of cyclosporine significantly reduce $\mathrm{CD} 8^{+} \mathrm{T}$ cell activity and can suppress acute rejection, ablates allograft vasculopathy. The occurrence of butylated hydroxytouline (V33) (Yu et al. 2012) in a significant amount can be accounted for its role in 


\section{S. Shrestha et al./Chemical Constituents.......}

hypercholesterolemia and atherosclerosis (Jilal \& Devraj 1996).

\section{Hepatoprotection}

There are several reports of the protective effect of $O$. sinensis extract for liver cirrhosis (Wang et al. 2008), hepatitis B (Zhou 1990, Wang et al. 2012), nonalcoholic fatty liver (Yang et al. 2007). Lu et al. (2011) reported polysaccharides from $O$. sinensis can decrease superoxide dismutase (SOD) activity and ameliorate local necrosis in liver.

\section{Hyperlipidemia / Hypercholesterolemia}

Kiho et al. (1996) reported CS-F30 (101) lowered the plasma triglyceride level and cholesterol level in mice. Koh et al. (2003) showed hot-water extract of $O$. sinensis can change very-low-density lipoprotein plus low-density lipoprotein (VLDL+LDL) and consequently decreased the atherogenic index and decreased blood serum cholesterol levels. The investigation of Yu et al. (2012) showed the presence of butylated hydroxytouline (BHT) $47.5 \%$ and $42.22 \%$ in mycelia culture by solid media and submerged fermentation, respectively. BHT (V33) is well known for inhibition of LDL-oxidation (Xiu et al. 1994), with $\mathrm{IC}_{50}$ value of $2.1 \mu \mathrm{M}$ (Shrestha et al. 2011). Presence of BHT in a significant amount can be reason of $O$. sinensis efficacy in treating hyperlipidemia and hypercholesterolemia (Jilal \& Devraj 1996). The study of Kim (2010) showed strong inhibition of cholesterol esterase with an $\mathrm{IC}_{50}$ value of $12.7 \mathrm{ig} / \mathrm{mL}$ by heteropolysaccharide PS-A (109), suggesting its use as a potential agent for control of hypercholesterolemia.

\section{Sexual dysfunction}

O. sinensis a trusted aphrodisiac, which enhances of libido and fertility in both sexes. Huang et al. (2004) reported steroidogenic enzyme expression in human granulosa-lutein cells (GLC). Ulibarri and Yahr (1997) observed cordycepin (27) treatment lead three times increased in lordosis quotients (LQs) and ovulation than in control. Further, Pao et al. (2012) reported cordycepin activated the phospholipase C/protein kinase C (PLC/PKC), but not PKA and PI3K, pathway to induce MA-10 cell steroidogenesis. Furthermore, Lew et al. (2011) showed stimulatory effects of cordycepin (27) on mouse leydig cell steroidognenesis. Nitric oxide (NO) has been well characterized as a mediator of inhibitory non-adrenergic non-cholinergic (NANC) neurotransmission in many organs including the urogenital system (Rand \& Li 1995). There are a series of reactions involved in the erection of muscles, in which NO is utilized. NO is the principal vasodilator, it activates guanylyl cyclase, present in the cell membrane, resulting in an increase in the level of cyclic guanosine mono phosphate (cGMP) leading to the relaxation of smooth muscles of the corpora cavernousa allowing inflow of blood leading to tumescence in both sexes (Burnett et al. 1992, Cellek \& Moncada 1998, Kim et al. 2003, Bansal et al. 2010). $b$-Sitosterol (12) is known for reinforcing the cell viability as well as increase in NO production significantly (Zhang et al. 2011) and D-mannitol (112) also facilitates NO production (Ohkuma et al. 1998) and both are major components in $O$. sinensis (Yu et al. 2012), which can be the possible cause of arousal or treatment for erectile dysfunction by $O$. sinensis.

\section{Immunoresponse}

Holliday and Cleaver (2008) mentioned $O$. sinensis as an option for antiretroviral drug. Further, Cheung et al. (2009) reported an exo-polysaccharide, cordysinocan (108) induced the cell proliferation and the secretion of interleukin-2, interleukin-6 and interleukin-8 in cultured T-lymphocytes. Furthermore, the phosphorylation of extracellular signal-regulated kinases (ERK) was induced transiently by its treatment and increased the phagocytosis activity and the enzymatic activity of acid phosphatase which verify the important its role of cordysinocan (108) in triggering immune responses.

The unique assemblage of sterols, nucleosides and polysaccharides as major constituents in $O$. sinensis are responsible for its widely acclaimed therapeutic value. The standardization of $O$. sinensis in various regions of Nepal and identification of most potential sites is needed. Further research for culturing $O$. sinensis in selected wild habitat with the artificial infection as well as adjustment of temperature, humidity and reduction of natural predators of Hepialus larva is needed to ensure sustainable harvest as well as economic benefit. Winkler (2009) reported that revenue of District forest office in Dolpo showed the steady rise in collection, 2006 (148 kg), 2007 (241 kg), 2008 (773 kg) and 2009 (872 $\mathrm{kg})$. However, drastic decline collection this year should be taken as a wakeup call for urgent need management of collection from nature (Taggart 2012). Extensive research on metabolites from wild $O$. sinensis is still awaited as researches are focused on mycelium culture due to relatively high cost of wild specimen. 
Nepal Journal of Science and Technology Vol. 13, No. 1 (2012) 43-58

\section{Acknowledgements}

We thank Next-Generation Bio-Green 21 Program (No. PJ008020), Rural Development Administration, Republic of Korea for providing research facilities.

\section{References}

Babu, B. and J.T. Wu. 2008. Production of natural butylated hydroxytoluene as an antioxidant by freshwater phytoplankton. Journal of Phycology 44:1447-1454.

Bansal, P., V. Gupta, M.V. Acharya, H. Kaur, R. Banal and S. Sharma. 2010. Garlic-potential substitute to synthetic aphrodisiacs for erectile dysfunction. Journal of Pharmacy Research 3:3072-3074.

Baral, S.R. and P.P. Kurmi. 2006. A compendium of medicinal plants of Nepal. R. Sharma publisher, Kathmandu, Nepal. 534 pp.

Bok, J.W., L. Lermer, J. Chilton, H.G. Klingeman and G.H.N. Towers. 1999. Antitumor sterols from the mycelia of Cordyceps sinensis. Phytochemistry 51:891-898.

Burnett, A.L., C.J. Lowenstein, D.S. Bredt, T.C.K. Chang and S.H. Snyder. 1992. Nitric oxide: a physiological mediator of penile erection. Science 257:401-403.

Cellek, S. and S. Moncada. 1998. Nitrergic neurotransmission mediates the non-adrenergic non-cholinergic responses in the clitoral corpus cavernosum of the rabbit. British Journal of Pharmacology 125:1627-1629.

Chen, M., F.W.K. Cheung, C.M. Hung, P.H. Kwan, S.P. Ip., L.Y. Hin., C.T. Che and L.W. Keung. 2012. Protective roles of Cordyceps on lung fibrosis in cellular and rat models. Journal of Ethnopharmacology. ( http://www.sciencedirect.com/science/article/pii/ S0378874112004333)

Chen, Y.Q., B. Hu, F. Xu, W. H. Zhang, H. Zhou and L.H. Qu. 2004. Genetic variation of Cordyceps sinensis, a fruit-body-producing entomopathogenic species from different geographical regions in China. Federation of European Microbiological Societies Microbiology Letters 230:153-158.

Cheung, J.K.H., J. Li, A.W.H. Cheung, Y. Zhu, K.Y.Z. Zheng, C.W.C. Bi, R. Ducan, R.C.Y. Choi, D.T.W. Lau and T.T.X. Dong. 2009. Cordysinocan, a polysaccharide isolated from cultured Cordyceps, activates immune responses in cultured T-lymphocytes and macrophages: signaling cascade and induction of cytokines. Journal of Ethno pharmacology 124: 61-68.

Devkota, S. 2006. Yarsagumba [Cordyceps sinensis (Berk.) Sacc.]; traditional utilization in Dolpa district, Western Nepal. Our Nature 4:48-52.

Ding, C., P.X. Tian, W. Xue, X. Ding, H. Yan, X. Pan, X. Feng, H. Xiang, H. Jun and X. Tian. 2011. Efficacy of Cordyceps sinensis in long term treatment of renal transplant patients. Frontiers in Bioscience E3: 301-307.

Diniz, R.O., L.K. Garla, J.M. Schneedorf and J.C.T. Carvalho. 2003. Study of anti-inflammatory activity of Tibetan mushroom, a symbiotic culture of bacteria and fungi encapsulated into a polysaccharide matrix. Pharmacological Research 47:49-52.

Dong, C. and Y. Yao. 2012. Isolation, characterization of melanin derived from Ophiocordyceps sinensis, an entomogenous fungus endemic to the Tibetan plateau. Journal of Bioscience and Bioengineering 113:474479.

Fu, H., W. Fu, M. Sun, Q. Shou, Y. Zhai, H. Cheng, L. Teng, X. Mou, Y. Li, S. Wan, S. Zhang, Q. Xu, X. Zhang, J. Wang, J. Zhu, X. Wang, X. Xu, G. Lv, J. Jin, W. Guo and Y. Ke. 2011. Kinetic cellular phenotypic profiling: prediction, identification, and analysis of bioactive natural products. Analytical Chemistry 83:6518-6526.

Gewali, M.B. 2008. Aspects of traditional medicine in Nepal. (Ed. S. Awale), Institute of Natural Medicine, University of Toyama, Japan, 175 p.

Giove, C. 2011. New Yorkers paying $\$ 800$ an ounce for worms that promise sexual prowess. NY Post, Jan 16. (http://www.nypost.com/p/news/local/ arousing_success_for_love_bug_p4CC6laeuVGjIQ4Q7xdPTK)

Gong, M., Q. Zhu, Q. Wang, T. Wang, X.L.Wang, J.X. Ma and W.J. Zhang. 1990. Molecular structure and immunoactivity of polysaccharide from Cordyceps sinensis (Berk) Sacc. Chinese Journal of Biochemistry and Molecular Biology 6:486-492.

Guan, J., F.Q. Yang and S.P. Li. 2010. Evaluation of carbohydrates in natural and cultured Cordyceps by pressurized liquid extraction and gas chromatography coupled with mass spectrometry. Molecules 15:42274241.

Guan, Y.J., G. Hu, M. Hou, H. Jiang, X. Wang and C. Zhang. 1992. Effect of Cordyceps sinensis on T-lymphocyte subsets in chronic renal failure. Chinese Journal of Integrative Medicine 12:338-339.

Guo, Q.C. and C. Zhang 1995. Clinical observations of adjunctive treatment of 20 diabetic patients with JinShuiBao capsule. Journal of Administration of Traditional Chinese Medicine 5:22.

Holliday, J. and M. Cleaver. 2008. Medicinal value of caterpillar fungi species of the Genus Cordyceps (Fr.) (Ascomycetes): A review. International Journal of Medicinal Mushrooms 10: 219-234.

Holliday, J., M. Cleaver and S.P. Wasser. 2005. Cordyceps. In: Encyclopedia of dietary supplements (Eds. P.M. Coats, M.R. Blackman, G. Cragg, M. Levine, J. Moss \& J White). Marcel Decker, USA. Pp. 1-13.

Huang, B.M., K.Y. Hsiao, P.C. Chuang, M.H. Wu, H.A. Pan and S.J. Tsai. 2004. Upregulation of steroidogenic enzymes and ovarian 17â-estradiol in human granulosa- 
lutein cells by Cordyceps sinensis mycelium. Biology of Reproduction 70:1358-1364.

Huang, L.F., Y.Z. Liang, F.Q. Guo, Z.F. Zhou and B.M. Cheng. 2003. Simultaneous separation and determination of active components of Cordyceps sinensis and Cordyceps militarris by LC/ESI-MS. Journal of Pharmaceutical and Biomedical Analysis 33:1155-1162.

Huang, Y., J. Lu, B. Zhu, Q. Wen, F. Jia, S. Zheng, T. Chen, Y. Li, G. Cheng and Z. Yi. 1987. Toxicity study of fermentation - Cordyceps mycelia B414. Zhongchengyao 10:24-25.

Jia, J.M., H.H. Tao and B.M. Feng. 2009. Cordyceamides A and B from the culture liquid of Cordyceps sinensis (Berk.) Sacc. Chemical \& Pharmaceutical Bulletin 57: 99-101.

Jia, J.M., X.C. Ma, C.F. Wu, L.J. Wu and G.S. Hu. 2005. Cordycedipeptide A, a new cyclodipeptide from the culture liquid of Cordyceps sinensis (Berk.) Sacc. Chemical \& Pharmaceutical Bulletin 53:582-583.

Jialal, I. and S. Devaraj. 1996. Low-density lipoprotein oxidation, antioxidants, and atherosclerosis: a clinical biochemistry perspective. Clinical Chemistry 42: 498506.

Jordan, J.L., A.M. Sullivan and T.D.G. Lee. 2008. C. sinensis ablates allograft vasculopathy when used as an adjuvant therapy with cyclosporine A. Transplant Immunology 19:159-166.

Jordan, J.L., A.M. Sullivan and T.D.G. Lee. 2008. Immune activation by a sterile aqueous extract of Cordyceps sinensis: mechanism of action. Immunopharmacology and Immunotoxicology 30:53-70.

Kadota, S., T. Shima and T. Kikuchi. 1986. Steroidal components of I-Tiam-Hong and Cordyceps sinensis, separation and identification by high performance liquid chromatography. Yakugaku Zasshi 106:10921097.

Kiho, T., A. Yamane, J. Hui, S. Usui and S. Ukai. 1996. Polysaccharides in fungi, XXXVI. Hypoglycemic activity of a polysaccharide (CS-F30) from the cultural mycelium of Cordyceps sinensis and its effect on glucose metabolism in mouse liver. Biological and Pharmaceutical Bulletin 19:294-296.

Kiho, T., H. Tabata and S. Ukai. 1986. A minor, proteincontaining galactomannan from a sodium carbonate extract of Cordyceps sinensis. Carbohydrate Research 156:189-197.

Kiho, T., J. Hui, A. Yamane and S. Ukai. 1993. Polysaccharides in fungi. XXXII. Hypoglycemic activity and chemical properties of a polysaccharide from the cultural mycelium of Cordyceps sinensis. Biological and Pharmaceutical Bulletin 16: 1291-1293.

Kiho, T., K. Ookubu, S. Usui, S. Ukai and K. Hirano. 1999. Structural features and hypoglycemic activity of a polysaccharide (CS-F10) from cultured mycelium of Cordyceps sinensis. Biological and Pharmaceutical Bulletin 22: 966-970.

Kim, G.H., B. Shrestha, S.Y. Lim, D.H. Yoon, W.C. Chang, D.J. Shin, S.K. Han, S.M. Park, J.H. Park, J.M. Sung, Y. Jang, N. Chung, K.C. Hwang and T.W. Kim. 2006. Cordycepin inhibits lipopolysaccharide-induced inflammation by the suppression of NF-êB through Akt and p38 inhibition in RAW 264.7 macrophage cells. European Journal of Pharmacology 545: 192199.

Kim, S.D. 2010. Isolation, structure and cholesterol esterase inhibitory activity of a polysaccharide, PS-A, from Cordyceps sinensis. Journal of the Korean Society for Applied Biological Chemistry 53:784-789.

Kim, S.W., S.J. Jeong, R. Munarriz, N.N. Kim, I. Goldstein and A.M. Traish. 2003. Role of the nitric oxide-cyclic GMP pathway in regulation of vaginal blood flow. International Journal of Impotence Research 15: 355361.

Koh, J.H., J.M. Kim, U.J. Chang and H.J. Suh. 2003. Hypochlesterolemic effect showed hot-water extract of mycelia of Cordyceps sinensis. Biological \& Pharmaceutical Bulletin 26: 84-87.

Kuo, Y.C., W.J. Tsai, J.Y. Wang, S.C. Chang, C.Y. Lind and M.S. Shiao. 2001. Regulation of bronchoalveolar lavage fluids cell function by the immunomodulatory agents from Cordyceps sinensis. Life Sciences 68: 1067-1082.

Kuo, Y.C., W.J. Tsai, M.S. Shiao, C.F. Chen and C.Y. Lin. 1996. Cordyceps sinensis as an immunomodulatory agent. American Journal of Chinese Medicine 24: 111125.

Lew, S.F., S.L. Poon, H.Y. Pao and B. Miin. 2011. The in vivo and in vitro stimulatory effects of cordycepin on mouse Leydig cell steroidognenesis. Bioscience, Biotechnology, and Biochemistry 75: 723-731.

Li, J., J. Lee, L. Xu, S. Yang and Z. Zou. 2003. Studies chemical constituents of Cordyceps sinensis (Berk) Sacc. Zhongguo Yaoxue Zazhi 38:499-501.

Li, S.P., F.Q. Yang and K.W.K. Tsim. 2006. Quality control of Cordyceps sinensis, a valued traditional Chinese medicine. Journal of Pharmaceutical and Biomedical Analysis 41: 1571-1584.

Li, S.P., G.H. Zhang, Q. Zeng, Z.G. Huang, Y.T. Wang, T.T.X. Dong and K.W.K. Tsim. 2006. Hypoglycemic activity of polysaccharide, with antioxidation, isolated from cultured Cordyceps mycelia. Phytomedicine 13: 428433.

Li, S.P., K.J. Zhao, Z.N. Ji, Song, Z.H., T.T.X. Dong, C.K. Lo, J.K.H. Cheung, S.Q. Zhu and K.W.K. Tsim. 2003. A polysaccharide isolated from Cordyceps sinensis, a traditional Chinese medicine, protects PC12 cells against hydrogen peroxide-induced injury. Life Sciences 73: 2503-2513. 
Li, S.P., Z.R. Su, T.T.X. Dong and K.W.K. Tsim. 2002. The fruiting body and its caterpillar host of Cordyceps sinensis show close resemblance in main constituents and anti-oxidation activity. Phytomedicine 9: 319-234.

Li, T.X. and J.S. Yang, 2009. A review and prospect on the studies of Cordyceps sinensis (Berk.) Sacc. Chinese Journal of Medical Guide 11: 1310-1316.

Lie, M.H., Q.X. Zhang, M.S. Zhu, X.Y. Liang and S.Q. Li. 2005. A study of acute and subchronic toxicity of Cordyceps sinensis polysaccharides in oral liquids. Modern Preventive Medicine 32: 1062-1063.

Lin, C.Y. 2002. (24R)-ergosta-7,22-dien-3â,5á,6â-triol from Cordyceps sinensis for improving kidney function in renal diseases. Patent JP 2002053488 A 20020219.

Lin, C.Y., C.C. Lin, C.C. Chen and Y.C. Kuo. 2004. The isolation of active fraction and active compound from Cordyceps sinensis can be used to improve bronchial hyper-responsiveness. Patent TW 582999 B 20040411.

Lin, C.Y., F.M. Ku, Y.C. Kuo, C.F. Chen, W.P. Chen, A. Chen and M.S. Shiao. 1999. Inhibition of activated human mesangial cell proliferation by the natural product of Cordyceps sinensis (H1-A): an implication for treatment of IgA mesangial nephropathy. Journal of Laboratory and Clinical Medicine 133: 55-63.

Liu, Y., F. Xu, B. Chen, J. Zhang and S. Yao. 2010. CZE determination of nucleosides and nucleobases from natural Cordyceps sinensis and cultured Cordyceps militaris. Yaowu Fenxi Zazhi 30: 24-29.

Lu, Y., X. Qiu, X. Liu, J. Zhu, X. Feng and Y. Zhang. 2011. Protective effect of polysaccharides from different parts of cultured Cordyceps sinensis on $\mathrm{CCl}_{4}$-induced liver damage in mice. Shipin Кехие 32: 319-322.

Nakamura, K., K. Konoha, Y. Yamaguchi, S. Kagota, K. Shinozuka and M. Kunitomo. 2003. Combined effects of Cordyceps sinensis and methotrexate on hematogenic lung metastasis in mice. Receptors Channels 9: 329-34.

Ohkuma, S., M. Katsura, Y. Hibino, A. Hara, K. Shirotani, E. Ishikawa and K. Kuriyama. 1998. Mechanisms for facilitation of nitric oxide-evoked [3H]GABA release by removal of hydroxyl radical. Journal of Neurochemistry 71:1501-1510.

Pao, H.Y., B.S. Pan, S.F. Leu and B.M. Huang. 2012. Cordycepin stimulated steroidogenesis in MA-10 mouse leydig tumor cells through the protein kinase C pathway. Journal of Agricultural and Food Chemistry 60: 4905-4913.

Qian, G.M., G.F. Pan and J.Y. Guo. 2012. Anti-inflammatory and antinociceptive effects of cordymin, a peptide purified from the medicinal mushroom Cordyceps sinensis. Natural Product Research (DOI:10.1080/ 14786419.2012.658800).

Rand, M.J. and C.G. Li. 1995. Nitric oxide in the autonomic and enteric nervous system. In: Nitric oxide in the nervous system (Ed. S. Vincent). Academic Press, USA. Pp. 227- 279.

Rao Y.K., S.H. Fang and Y.M. Tzeng. 2007. Evaluation of the anti-inflammatory and anti-proliferation tumoral cells activities of Antrodia camphorata, Cordyceps sinensis, and Cinnamomum osmophloeum bark extracts. Journal of Ethnopharmacology 114:78-85.

Shahed, A.R., S.I. Kim and D.A. Shoskes. 2001. Downregulation of apoptotic and inflammatory genes by Cordyceps sinensis extract in rat kidney following ischemia/reperfusion. Transplantation Proceedings 33: 2986-2987.

Shen, Q.P., G.H. Qin and L.F. He. 2001. Toxicity and mutation of $C$. sinensis polysaccharide powder fed orally. Teratogenesis Carcinogenesis and Mutagenesis 13: 179-181.

Shiao, M.S., Z.N. Wang, L.J. Lin, J.Y. Lien and J.J. Wang. 1994. Profiles of nucleolsides and nitrogen bases in Chinese medicinal fungus Cordyceps sinensis and related species. Botanical Bulletin Academica Sinica 35: 261-267.

Shrestha, B. 2010. Yarsagumba ko kheti (Cultivation of Yarsagumba). Bigyan Lekhmala 358: 4-11 (in Nepali).

Shrestha, B. 2011. Diversity of Cordyceps fungi in Nepal. Nepal Journal of Science and Technology 12:103-110.

Shrestha, B., W.M. Zhang, Y.J. Zhang and X.Z. Liu. 2010. What is Chinese caterpillar fungus Ophiocordyceps sinensis (Ophiocordycipitaceae)? Mycology 1: 228236.

Shrestha, T.K., S. Shrestha and A.K. Shrestha. 2011. Himalayan flowers, trees and animals. B. Shrestha publisher, Kathmandu, Nepal, 160 p.

Shrestha, S., J.H. Park, D.Y. Lee, J.G. Cho, D.G. Lee, M.H. Cho, T.S. Jeong, H.C. Kang and N.I. Baek. 2011. Inhibition of low density lipoprotein-oxidation, ACAT1, and ACAT-2 by lignans from the bark of Machilus thunbergii. Journal of the Applied Biological Chemistry 54: 63-66.

Sung, G.H., N.L. Hywel-Jones, J.M. Sung, J.J. Luangsaard, B. Shrestha and J.W. Spatafora. 2007. Phylogenetic classification of Cordyceps and clavicipitaceous fungi. Studies in Mycology 57: 5-59.

Taggart, F. 2012. Nepal 'Himalayan Viagra' harvest drops to record low. Agence France-Presse (AFP), July 10. http://www.mysinchew.com/node/74283.

Ulibarri, C. and P. Yahr. 1987. Poly-A+ mRNA and defeminization of sexual behavior and gonadotropin secretion in rats. Physiology \& Behavior 39: 767-774.

Wang, J., Y.M. Liu, W. Cao, K.W. Yao, Z.Q. Liu and J.U. Guo. 2012. Anti-inflammation and antioxidant effect of Cordymin, a peptide purified from the medicinal mushroom Cordyceps sinensis, in middle cerebral artery occlusion-induced focal cerebral ischemia in rats. 
Metabolic Brain Disease 27: 159-165.

Wang, S., F.G. Yang, K. Feng, D.G. Li, J. Zhao and S.P. Li. 2009. Simultaneous determination of nucleosides. Journal of Separation Science 32: 4069-4076.

Wang, X.B., P. Liu, Z.P. Tang, F.H. Li, C.H. Liu, Y.Y. Hu and L.M. Xu. 2008. Cordyceps mycelia extract decreases portal hypertension in rats with dimethylnitrosamine-induced liver cirrhosis: a study on its histological basis. Journal of Chinese Integrative Medicine 6: 1136-1144.

Wang, X.B., Y.Y. Jiang and C.Y. Zhao. 2012. Clinical research of xinganbao capsule on the treatment of chronic hepatitis B liver fibrosis. Chinese Journal of Integrated Traditional and Western Medicine 32: 325-328.

Wang, X.L. and Y.J. Yao. 2011. Host insect species of Ophiocordyceps sinensis: a review. ZooKeys 127: 43-59.

Wang, Y., M. Wang, Y. Ling, W. Fan, Y. Wang and H. Yin. 2009. Structural determination and antioxidant activity of a polysaccharide from the fruiting bodies of cultured Cordyceps sinensis. American Journal of Chinese Medicine 37: 977-989.

Wasser, S.P. 2002. Medicinal mushrooms as a source of antitumor and immunomodulating polysaccharides. Applied Microbiology and Biotechnology 60: 258-274.

Wei, W., H. Chen, G.R. Zhang, S.P. Li, Q.Y. Peng, X. Zhong and X. Liu. 2011. Molecular identification and food source inference of constructive plants, native to the Ophiocordyceps sinensis habitat. African Journal of Biotechnology 10:159-167.

Winkler, D. 2009. Caterpillar fungus (Ophiocordyceps sinensis) production and sustainability on the Tibetan plateau in the Himalayas. Asian Medicine 5:291-316.

Wu, Y., C. Sun and Y. Pan. 2006. Studies on isolation and structural features of a polysaccharide from the mycelium of a Chinese edible fungus (Cordyceps sinensis). Carbohydrate Polymers 63: 251-256.

Wu, Y., N. Hu, Y. Pan, L. Zhou and X. Zhou. 2007. Isolation and characterization of a mannoglucan from edible Cordyceps sinensis mycelium. Carbohydrate Research 342: 870-875.

Xiao, L., Y. Ge, L. Sun, X.X. Xu, P. Xie, M. Zhan, M. Wang, Z. Dong, J. Li, S. Duan, F. Liu and P. Xiao. 2012. Cordycepin inhibits albumin-induced epithelialmesenchymal transition of renal tubular epithelial cells by reducing reactive oxygen species production. Free Radical Research 46: 174-183.

Xiu, R.J., A. Freyschuss, X. Ying, L. Berglund, P. Henriksson and I. Bjorkhem. 1994. The antioxidant butylated hydroxytoluene prevents early cholesterol-induced microcirculatory changes in rabbits. Journal of Clinical Investigation 93: 2732-2737.

Xu, F., J.B. Huang, L. Jiang, J. Xu and J. Mi. 1995. Amelioration of cyclosporin nephrotoxicity by
Cordyceps sinensis in kidney transplanted recipients. Nephrology Dialysis Transplantation 10: 142-143.

Yang, F.Q., K. Feng, J. Zhao and S.P. Li. 2009. Analysis of sterols and fatty acids in natural and cultured Cordyceps by one-step derivatization followed with gas chromatography-mass spectrometry. Journal of Pharmaceutical and Biomedical Analysis 49: 11721178.

Yang, M.L., P.C. Kuo, T.L. Hwang and T.S. Wu. 2011. Anti-inflammatory principles from Cordyceps sinensis. Journal of Natural Products 74: 1996-2000.

Yang, Z., D. Dai and W. Shen. 2007. Effects of Cordyceps sinensis and reduced glutathione on experimental mouse model with non-alcoholic fatty liver disease. Journal of the Third Military Academy 29: 2176-2178.

Yoshida, J., S. Takamura, N. Yamaguchi, L.J. Ren, H. Chen, S. Koshimura and S. Suzuki. 1989. Antitumor activity of an extract of Cordyceps sinensis (Berk.) Sacc. against murine tumor cell lines. Japanese Journal of Experimental Medicine 59: 157-161.

Yue, G.G.L, C.B.S. Lau, K.P. Fung, P.C. Leung and W.H. Ko. 2008. Effects of Cordyceps sinensis, Cordyceps militaris and their isolated compounds on ion transport in Calu-3 human airway epithelial cells. Journal of Ethnopharmacology 117: 92-101.

Yu, S., Z. Zhang and M. Fan. 2012. Analysis of volatile compounds of mycelia of Hirsutella sinenis, the anamorph of Ophiocordyceps sinensis. Applied Mechanics and Material 140: 253-257.

Yun, Y., S. Han, S. Lee, S.K. Ko, C.K. Lee, N.J. Ha and K. Kim. 2003. Anti-diabetic effects of CCCA, CMESS, and cordycepin from Cordyceps militaris and the immune responses in streptozotocin-induced diabetic mice. Natural Product Sciences 9: 291-298.

Zhang, J., J. Hao, J. Guo, B. He and H. Huang. 2011. Effect of fistular onion stalk extract on the level of NO and expression endothelial NO synthase (eNOS) in human umbilical vein endothelium cells. African Journal of Biotechnology 10: 2536-2540.

Zhang, J., Y. Yu, Z. Zhang, Y. Ding, X. Dai and Y. Li. 2011. Effect of polysaccharide from cultured Cordyceps sinensis on immune function and anti-oxidation activity of mice exposed to ${ }^{60} \mathrm{Co}$. International Immunopharmacology 11: 2251-2257.

Zhang, S.S., D.S. Zhang, T.J. Zhu and X.Y. Chen. 1991. A pharmacological analysis of the amino acid components of Cordyceps sinensis Sacc. Acta Pharmaceutica Sinica 26: 326-330.

Zhang, X., P. Geoffroy, M. Miesch, J.D. David, F. Raul, D. Aoude-Werner and E. Marchioni. 2005. Gram-scale chromatrogrphic purification of $b$-sitosterol synthesis and characterization of $b$-sitosterol oxides. Steroids 70: 886-895.

Zhao, Y.Y., X. Shen, X. Chao, C.C. Ho, X.L. Cheng, Y. 
Zhang, R.C. Lin, K.J. Du, W.J. Luo, J.Y. Chen and W.J. Sun. 2011. Ergosta-4,6,8(14),22-tetraen-3-one induces $\mathrm{G} 2 / \mathrm{M}$ cell cycle arrest and apoptosis in human hepatocellular carcinoma HepG2 cells. Biochimica et Biophysica Acta - General Subjects 1810: 384-390.

Zhao, Y.Y., X.L. Cheng, Y. Zhang, Y. Zhao, R.C. Lin and W.J. Sun. 2009. Simultaneous determination of eight major steroids from Polyporus umbellatus by high performance liquid chromatography coupled with mass spectrometry detections. Biomedical Chromatography 24: 222-230.

Zhong, S., H.J. Pan, L. Fan, G., G.Y. Lv, Y.Z. Wu, B. Parmeswaran, A. Pandey and C.R. Soccol. 2009. Advances in research of polysaccharides in Cordyceps species. Food Technology and Biotechnology 47: 304312.

Zhou, L., W. Yang, Y. Xu, Q. Zhu, Z. Ma, T. Zhu, X. Ge and J. Gao. 1990. Short-term curative effect of cultured Cordyceps sinensis (Berk.) Sacc. mycelia in chronic hepatitis B. China Journal of Chinese Materia Medica 15:53-55, 65.

Zhu, J.S., G.M. Halpern and K. Jones. 1998a. The scientific rediscovery of a precious ancient Chinese herbal regimen: Cordyceps sinensis: Part I. Journal of Alternative and Complementary Medicine 4: 289-303.

Zhu, J.S., G.M. Halpern and K. Jones. 1998b. The scientific rediscovery of a precious ancient Chinese herbal regimen: Cordyceps sinensis: Part II. Journal of Alternative and Complementary Medicine 4: 429-457. 